ORIGINAL ARTICLE

\title{
Anthropometry of young competitive sport rock climbers
}

\author{
P B Watts, L M Joubert, A K Lish, J D Mast, B Wilkins
}

Br J Sports Med 2003;37:420-424

See end of article for authors' affiliations

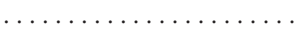

Correspondence to: Professor Watts

1401 Presque Isle Avenue,

Marquette, Ml 49855 ,

USA; pwatts@nmu.edu

Accepted

2 October 2002
Background: Adult elite competitive rock climbers are small in stature with low body mass and very low body fat percentage. These characteristics have generated concern that young climbers may attempt body mass reduction to extreme levels with adverse consequences for health and performance. No published anthropometry data for young competitive climbers exist.

Objective: To describe the general anthropometric characteristics of junior US competitive rock climbers.

Methods: Ninety subjects (mean (SD) age 13.5 (3.0) years) volunteered to participate. All competed at the Junior Competition Climbers Association US National Championship. Anthropometric variables, including height, mass, body mass index (BMI), arm span, biiliocristal and biacromial breadths, skinfold thickness at nine anatomical sites, forearm and hand volumes, and handgrip strength, were measured. Selected variables were expressed as ratio values and as normative age and sex matched centile scores where appropriate. A control group $(n=45)$ of non-climbing children and youths who participated in a variety of sports activities, including basketball, cross country running, cross country skiing, soccer, and swimming, underwent the same testing procedures in the Exercise Science Laboratory of Northern Michigan University.

Results: Mean (SD) self reported climbing ability was 11.80 (1.20), or about $5.11 \mathrm{~d}$ on the Yosemite decimal system scale. The mean (SD) experience level was $3.2(1.9)$ years, and subjects competed in 10 (5) organised competitions over a 12 month period. Despite similarity in age, there were significant differences $(p<0.01)$ between climbers and control subjects for height, mass, centile scores for height and mass, ratio of arm span to height ("ape index"), biiliocristal/biacromial ratio, sum of seven and sum of nine skinfolds, estimated body fat percentage, and handgrip/mass ratio. Despite significantly lower skinfold sums and estimated body fat percentage, no differences were found between climbers and controls for absolute BMI or BMl expressed as a centile score.

Conclusions: Young competitive climbers have similar general anthropometric characteristics to elite adult climbers. These include relatively small stature, low body mass, low sums of skinfolds, and high handgrip to mass ratio. Relative to age matched athletic non-climbers, climbers appear to be more linear in body type with narrow shoulders relative to hips. Differences in body composition exist between climbers and non-climbing athletes despite similar BMI values.
$\mathrm{T}$ he recreational activity of rock climbing continues to grow in popularity, and the proliferation of indoor climbing walls has fostered informal and formal competitive venues. Study of the climber as an athlete is a relatively new pursuit within the exercise and sport sciences, and early research has focused on physical characteristics that may be associated with high level performance. Watts et al found elite adult competitive climbers to be small in stature with low body mass and very low body fat percentages. Other studies that rated subjects as "elite" or "expert" have reported similar results. ${ }^{2-4}$ Several studies have reported relatively high hand grip strength/body mass ratios in high level climbers. ${ }^{15}$ Although they found high grip strength/mass ratios in elite climbers, Watts et al categorised absolute handgrip strength scores as "moderate" and suggested that the high strength/ mass ratios were due to the effect of low body mass.

The association of high level climbing performance with small stature and low body fat percentage, and the possibility of improving strength/mass ratio through body mass reduction, has raised concern about the potential long term health effects on climbers. Some authors have expressed concern over the effects of extreme body fat reduction, potential for eating disorders, and associated health and performance risks for climbers. ${ }^{67}$ Despite anecdotal references to eating disorders and unrealistic weight reduction practices in climbers, no supportive published data in this area are available and no data exist to link the pursuit of excellence in climbing with associated long term health risks.
Despite this lack of data, there has been at least one action to discourage manipulation of body mass through imposition of a minimum body mass index (BMI) standard on young competitive climbers. ${ }^{8}$ Whether the selected levels of limitation are justifiable or reasonable for this specialised group of athletes is not known because no published anthropometry data for young competitive sport climbers exist.

This study attempted to fill a portion of this information void relative to young climbers who perform at high levels. Specifically, its purpose was to evaluate anthropometry data for experienced junior competitive sport climbers between the ages of 9 and 18 years and to compare data for this group with an age matched group of physically active youth who do not climb.

\section{METHODS}

Ninety experienced competitive climbers ( 52 boys, 38 girls) volunteered and gave informed consent to participate in the study. These subjects were competitors at the United States National Championship of the Junior Competition Climbers Association (JCCA). All data for the climber group (climbers) were collected before competition at the site of the JCCA National Championship in September, 2000.

Abbreviations: BMI, body mass index; YDS, Yosemite decimal system 
At the time of data collection, climbers completed a questionnaire to document training volume and self reported climbing ability for best top rope (with the rope anchored above the climber at all times) and red point (where the climber clips the rope into intermediate anchors along the climbing route) ascents. Climbing ability was defined as the most difficult ascent by top rope or red point style, whichever was higher, rated on a modified Yosemite decimal system (YDS) scale. The modified YDS scale for technical "free" climbing difficulty, where no artificial means are used to aid progress, currently extends from 0 to 15 with letter grades of a, b, c, and d subdividing the numerical categories. Letter subdivisions were assigned values of $\mathrm{a}=0.00, \mathrm{~b}=0.25, \mathrm{c}=0.50$, and $d=0.75$. Thus, a YDS rating of $13 \mathrm{~b}$ became 13.25 for the calculation of means. A similar rating conversion has been previously used by Watts et al. ${ }^{1}$

A comparison group of 45 age matched volunteers (controls; 30 boys, 15 girls) gave informed consent and underwent the same testing procedures as the climbers. Controls consisted of youths who were physically active in one or more organised sports but did not participate in climbing. They came from a variety of sports and activities including basketball, cross country running, cross country skiing, soccer, and swimming. All control testing was carried out in the Exercise Science Laboratory of Northern Michigan University. Data were expressed in the same way as for climbers to enable comparisons between the two groups.

All anthropometry measurements were made in a resting state according to established procedures. ${ }^{9}$ A Lafayette anthropometer was used, by a single investigator, to obtain all skeletal dimensions, with measurements made to the nearest millimetre. Standing height was recorded to the nearest half centimetre with the subject barefoot and with the back against a vertical wall. Mass was measured using a Tanita calibrated electronic scale. BMI was calculated as mass/height ${ }^{2}$ where mass was expressed in kilograms and height in metres. Height, mass, and BMI were expressed as absolutes and as normative centile scores according to tables published by the US Department of Health and Human Services Centers for Disease Control and Prevention (CDC)..$^{10}$

Arm span was measured in the standing position with the arms abducted horizontally. The greatest tip to tip distance between the extended fingers was recorded in centimetres. The ratio of arm span to height, known to climbers as the ape index, was calculated as arm span divided by height.

Biiliocristal breadth was measured as the distance between the most lateral points on the iliac tubercles. Biacromial breadth was measured as the distance between the most lateral points on the acromion processes. Biiliocristal/ biacromial ratio was calculated as the biiliocristal breadth divided by the biacromial breadth. A lower score for this ratio indicates a more triangular torso.

Skinfold thickness was measured to the nearest $0.5 \mathrm{~mm}$ with a calibrated Lange caliper at nine anatomical sites by the same trained technician. The nine sites measured were chest, subscapula, midaxilla, suprailiac, iliac crest, abdomen, triceps, thigh, and calf. All measurements were taken on the subject's right side. Percentage body fat (\%fat) values were estimated by two different methods. The procedures of Jackson and Pollock ${ }^{11}$ use seven skinfold site measurements to estimate body density, with \%fat subsequently calculated by the Brozek equation. ${ }^{4}$ The sum of the seven skinfolds (S7) used in the Jackson-Pollock equations and the sum of all nine skinfolds (S9) were calculated as variables. \%fat was also estimated directly from two skinfold measurements (triceps and calf) by the youth specific equations of Slaughter et al. ${ }^{13}$

Forearm and hand volumes were measured to the nearest millilitre by water displacement with the arm in a vertical anatomical position. Hand volume was measured by immersion to the level of a horizontal line between the styloid processes of the radius and ulna. Hand-forearm volume was

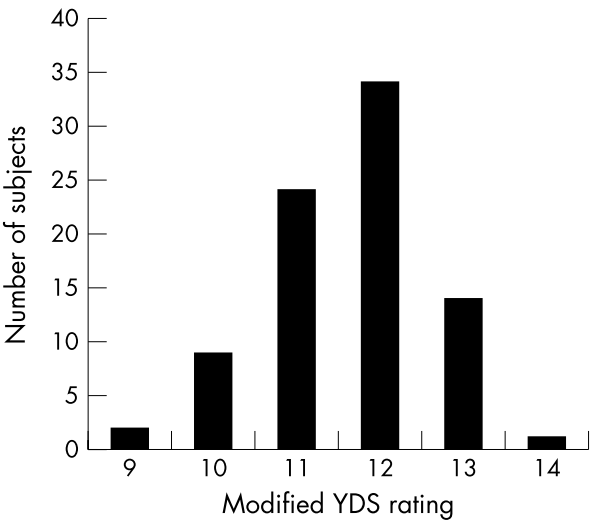

Figure 1 Distribution of climbing ability $(n=90)$. YDS, Yosemite decimal system.

measured by immersion to the level of the distal edge of the medial epicondyle of the humerus. Forearm volume was calculated as the difference between hand volume and handforearm volume.

An adjustable Jaymar hydraulic hand dynamometer was used to record handgrip force, with the subject seated and the elbow flexed to $90^{\circ}$. Maximum handgrip forces for each hand were recorded in kilograms as the highest of two trials. Right and left maximum handgrip forces were averaged to provide an average handgrip score. Handgrip/mass ratio was calculated as handgrip divided by total body mass.

Jandel Sigma Stat software was used for all statistical analyses. Means (SD) were calculated for measured and calculated variables. Analysis of variance with Tukey post hoc tests were used to test for differences between groups. A p level of 0.05 or less was considered significant for all analyses.

\section{RESULTS}

Mean ages were 13.5 (3.0) and 13.7 (2.7) years for climbers and controls respectively. Climbers had participated in the activity for 3.2 (1.9) years and had competed in 10 (5) organised competitions within a 12 month period.

The mean self reported climbing ability for climbers was rated at $11.80(1.20)$, or around $11 \mathrm{~d}$ on the modified Yosemite YDS scale. Figure 1 presents the overall distribution of abilities among the numerical divisions of the YDS rating system.

Table 1 presents means (SD) and minimum-maximum ranges for selected variables of training volume reported by the group of climbers. Control subjects reported a mean sport participation frequency of more than 100 sessions a year and a mean practice/competition time of 7.8 (4.5) hours a week in season. Control subjects also reported a mean of 6.5 (7.0) hours a week of aerobic type exercise as part of or in addition to sports participation.

Table 2 presents means (SD) for selected measured variables. Significant differences between climbers and controls are indicated. The wide range of ages in our subjects and consequent variability in growth and developmental stages

\begin{tabular}{lll}
$\begin{array}{l}\text { Table } 1 \\
(\mathrm{n}=90)\end{array}$ & Variables of training volume for climbers \\
\hline Variable & Mean (SD) & $\begin{array}{l}\text { Range } \\
\text { (min-max) }\end{array}$ \\
\hline Climbing season (months/year) & $9.6(2.9)$ & $3.0-12.0$ \\
Climbing sessions (days/week) & $3.5(1.2)$ & $1.0-7.0$ \\
Climbing duration (hours/session) & $3.3(1.5)$ & $1.0-10.0$ \\
Aerobic exercise (hours/week) & $5.9(5.2)$ & $0.0-24.5$ \\
\hline
\end{tabular}


Table 2 Means (SD) for selected anthropometric variables of the sample

\begin{tabular}{|c|c|c|c|c|}
\hline Variable & Controls $(n=45)$ & Climbers $(n=90)$ & Male climbers $(n=52)$ & Female climbers $(n=38)$ \\
\hline Height $(\mathrm{cm})$ & 167.1 (14.0) & $158.5(15.2)^{*}$ & $162.2(15.6)$ & $151.3(11.9)$ \\
\hline Height percentile & $79.3(25.3)$ & $50.0(28.7)^{*}$ & $44.8(26.0)$ & $54.4(31.3)$ \\
\hline Mass $(\mathrm{kg})$ & $54.1(15.0)$ & $47.8(13.4)^{*}$ & $51.5(13.6)$ & $40.6(9.6)$ \\
\hline Mass percentile & $57.8(25.6)$ & $39.4(23.5)^{*}$ & $40.8(23.6)$ & $39.8(24.6)$ \\
\hline Height/mass ratio & $3.28(0.78)$ & $3.51(0.74) \dagger$ & $3.33(0.73)$ & $3.86(0.64)$ \\
\hline BMI & $19.0(3.2)$ & $18.6(2.3)$ & $19.1(2.2)$ & $17.5(2.1)$ \\
\hline BMI percentile & 38.7 (29.7) & 32.7 (21.5) & $36.9(21.3)$ & $29.2(21.6)$ \\
\hline Ape index & $0.95(0.15)$ & $1.01(0.02)^{*}$ & $1.02(0.02)$ & 1.01 (0.02) \\
\hline Biliocrist/biacrom ratio & $0.74(0.05)$ & $0.86(0.08)^{*}$ & $0.87(0.08)$ & $0.86(0.08)$ \\
\hline$\Sigma 7$ skinfolds (mm) & $76.7(33.4)$ & $50.4(14.5)^{*}$ & $45.3(13.0)$ & $56.0(14.5)$ \\
\hline$\Sigma 9$ skinfolds (mm) & $101.3(45.2)$ & $66.5(20.5)^{*}$ & $59.3(19.2)$ & 74.3 (19.6) \\
\hline \%Fat Jackson-Pollock & $11.3(6.6)$ & $7.8(4.4)^{*}$ & $4.4(2.2)$ & $12.2(2.6)$ \\
\hline \%Fat Slaughter & 18.7 (6.9) & $13.0(3.7)^{*}$ & $11.0(2.8)$ & $15.9(2.9)$ \\
\hline Forearm volume (ml) & $824.7(266.6)$ & $828.8(370.1)$ & $903.9(254.8)$ & $671.7(190.6)$ \\
\hline Forearm+hand volume (ml) & $1148.9(426.8)$ & $1116.4(344.9)$ & $1234.4(326.8)$ & $902.0(235.3)$ \\
\hline Average HG $(\mathrm{kg})$ & $30.7(13.4)$ & $32.8(12.8)$ & $36.5(12.9)$ & $25.1(6.8)$ \\
\hline HG/mass ratio & $0.55(0.13)$ & $0.67(0.12)^{*}$ & $0.70(0.13)$ & $0.62(0.08)$ \\
\hline
\end{tabular}

make comparison and discussion of absolute data difficult. For this reason we have not compared boys with girls, although these data are presented in the right two columns of table 2 . We have also used centile scores and ratio variables when possible.

Climbers were significantly smaller in stature and had a lower body mass than the controls $(\mathrm{p}<0.01$; table 2$)$. These significant differences were also evident when data were expressed as normative centile scores $(\mathrm{p}<0.01$; table 2$)$. Height/mass ratio was also higher for climbers $(p<0.05$; table 2 ); however, BMI did not differ between the groups. Mean centile scores for BMI were lower for climbers, but this difference was not significant $(\mathrm{p}=0.09)$.

Both S7 and S9 skinfold measures were significantly lower for climbers, as were the results of both \%fat estimations. The Slaughter equations yielded significantly higher mean \%fat estimations than the Jackson-Pollock equations $(p<0.01)$. Figure 2 presents means (SD) for individual skinfold site measurements by group and sex. Mean values were significantly higher for female than male climbers at the triceps, thigh, and calf sites. Mean values were significantly higher for female than male controls at the thigh and calf sites. Skinfold thicknesses were significantly lower for climbers than controls at the chest, iliac crest, abdomen, triceps, thigh, and calf for girls and at the chest, suprailiac, iliac crest, abdomen, triceps, thigh, and calf for boys.

Forearm volume, hand +forearm volume, and handgrip force were not significantly different between climbers and controls. The handgrip/mass ratio was significantly higher in climbers.

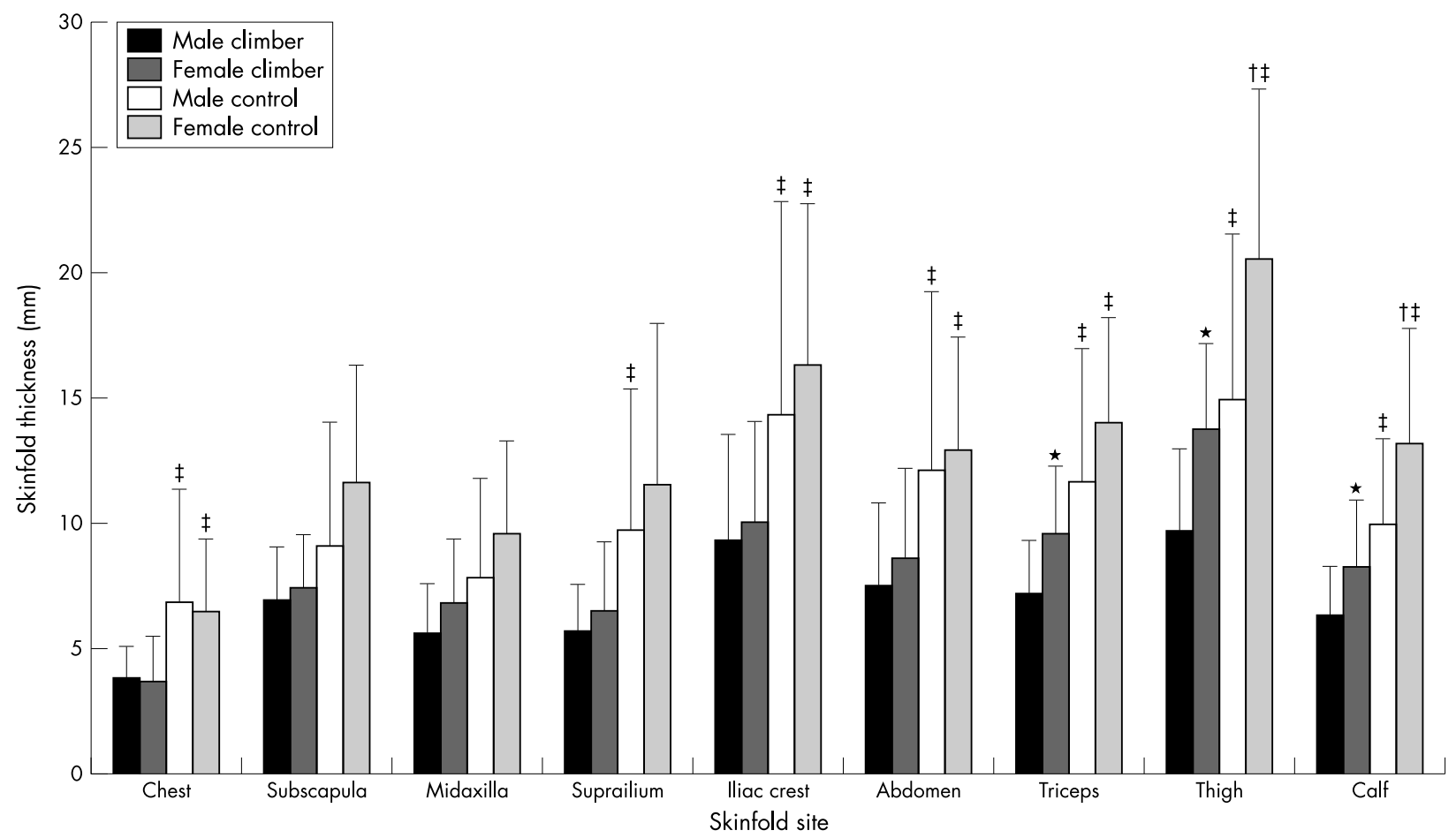

Figure 2 Means (SEM) for individual skinfold measurements by group and sex. *Significantly different from male climbers; $p<0.05$. $\dagger$ Significantly different from male controls; $p<0.05$. ¥Significantly different from same sex climbers; $p<0.05$. 


\section{DISCUSSION}

Competitive climbing has become increasingly popular among children and youth in recent years. The US Competition Climbing Association (formerly the JCCA) sanctioned over 100 competitions for the year 2002. The increased participation of children and youth in competitive climbing highlights the popularity of this sport and increases the interest in research examining this unique population. However, recent published research has focused solely on adult climbers. ${ }^{1-4}$ 14-19 $^{19}$ This study presents the first large sample descriptive data for young competitive climbers.

The mean ability level of the group of climbers in this study was $11.80(1.20)$ on the modified YDS scale or around $5.11 \mathrm{~d}$ with the median at 5.12a on the normal YDS scale. The mean ability for this group of young climbers is lower than previously reported mean abilities of elite adult climbers. Watts et al $^{1}$ reported mean ability levels of $5.13 \mathrm{C}$ and $5.12 \mathrm{C}$ respectively for male $(n=21)$ and female $(n=18)$ semifinalists at a 1989 International World Cup competition. The adult subjects of Watts et al, however, were older (men $=26.6$ (4.2) years; women $=27.8$ (2.0) years) and had more climbing experience $($ men $=11.2(4.9)$ years; women $=8.8$ (3.7) years) than the present subjects. Figure 1 illustrates that a large number of these young climbers perform at a comparatively high standard. This is notable considering the large age and experience differences between the present sample and the adult climbers of a decade ago.

As in previous studies with adult climbers, the young climbers in this study were found to be relatively small in stature and had low body mass, with means at or below the 50th centile for age and sex matched norms. Climbers were also significantly smaller and lighter than athletic control subjects. The small stature and low body mass in climbers minimises the work requirement of movement along the climbing route. A lighter mass also reduces the force output in muscle that would be required to sustain position and maintain a given hand configuration. This could result in a slower rate of fatigue in smaller climbers than their heavier counterparts.

A concern that young climbers may manipulate body mass to extremes in attempts to gain advantage has led one organisation to adopt a set of minimum BMI standards for competitive climbers. ${ }^{8}$ The ÖSK BMI limits vary from 16.00 to 17.00 for girls and from 17.00 to 18.00 for boys between 14 and 18 years of age. We applied these standards to our data and found that 21 of the 90 climbers, or $23.3 \%$, would not have passed the BMI cut off standards of ÖSK. All of these 21 climbers were under 16 years of age. Although this seems like a high percentage, it should be noted that 11 of the 45 control athletes, or $24.4 \%$, would not have passed the ÖSK BMI cut offs. We also found no significant differences between climbers and controls for absolute BMI score or for BMI expressed as a centile score.

Despite no BMI related differences between climbers and controls, we did find significant differences for \%fat by either estimation method. Our data also indicate that large discrepancies between equations for \%fat estimation can occur. The Brozek equation may overestimate \%fat in children because it is based on data from older adult cadavers. ${ }^{20}$ Still, for our subjects, the Brozek equation yielded lower \%fat values than the Slaughter equation. Unresolved questions about the assumptions of tissue densities for children and adolescents, particularly in athletic youth, discourage the application of body fat estimation equations in these populations.

Although interpretation of the \%fat data is problematic, large differences between the groups were observed for the S7 and S9 skinfold measures. It is also notable that both male and female climbers had lower individual site skinfold means at several sites than their respective controls (fig 2). Watts et al ${ }^{1}$ compared individual skinfolds at seven sites between male and female finalists at an international climbing competition and found mean thicknesses for female climbers to exceed values for male climbers at only two sites, the triceps and thigh. The current data for junior climbers differ in this respect, with means for female climbers exceeding those for male climbers at all sites except the chest (fig 2), although these differences were only significant for the triceps, thigh, and calf.

The differences in skinfold sums between climbers and controls indicate that the climbers had relatively less body fat. The S7 skinfold means for male and female climbers were 45.3 (13.0) and 56.0 (14.5) mm respectively. Although these values are significantly lower than the controls, they do not necessarily indicate severely low body fat levels. The S7 skinfold values for our subjects are higher than data previously reported for elite adult male (37.8 (6.8) $\mathrm{mm})$ and female $(42.5(8.9) \mathrm{mm})$ competitive climbers. ${ }^{1}$ Sum of skinfold values tend to increase from a point just after puberty to a peak at about 50 years of age. ${ }^{20}$ Whether these athletes will follow a reverse of this trend and exhibit the very low body fat levels that have been observed in elite adult climbers will require longitudinal data.

Considering the equivalent BMI for climbers and controls and the significant differences in skinfold measures, the climbers appear to be proportionately heavier in lean mass and lower in fat mass than the controls. The fact that the climbers had significantly lower skinfold sums than non-climbers without a difference in BMI suggests that BMI scores are inappropriate for screening subjects for extreme reductions in body weight and body fat. This is supported by Deurenberg et $a l^{21}$ who found low correlations between BMI and \%fat in a large group of subjects aged 7-20 years. Further study in this area is needed.

Possession of a long reach relative to height is thought to have a positive influence on climbing performance. Climbers describe the ratio of arm span to height as an ape index and place significance on values over 1.00. The climbers in this study had significantly higher ape index scores than the controls. Values were similar to those observed by Mermier, et al ${ }^{18}$ for adult male (1.00-1.08) and female (0.96-1.11) climbers. However, there was no correlation between ape index and climbing ability in our subjects, with $r=0.05$. This low correlation is probably due to the relatively small variability in ape index among the climbers ( $\mathrm{SD}=0.02)$. It is also possible that the ape index becomes more important when other traits are equivalent.

The higher biiliocristal/biacromial ratio in climbers indicates a less triangular torso than the controls. Most of this difference is accounted for by a narrower biacromial breadth (28.1 (2.5) $v 35.7(4.1) \mathrm{mm}$ ) relative to biiliocristal breadth (24.1 (2.6) $v 26.2(2.6) \mathrm{mm})$ in climbers than controls respectively. This narrower shoulder structure in the climbers could account for a portion of the lower mass observed. Perhaps of more significance is the possibility of a longer arm structure in climbers. The narrow biacromial breadth combined with the relatively large ape index suggests that the climbers had a longer arm component of the total finger tip to tip span distance than the controls. This could have implications for reach distance for a given body position and may be a more important factor than ape index alone.

The lack of a significant difference between climbers and controls for handgrip force is supported by our earlier research, which found absolute handgrip strength in elite climbers to rank between the 50th and 75th centiles for age and sex matched norms. ${ }^{1}$ When handgrip strength was expressed relative to body mass, as handgrip/mass ratio, climbers rated significantly higher than controls. Scores for boys $(0.70(0.13))$ and girls $(0.62(0.08))$ in this study compare closely with reported values for elite adult male $(0.78(0.06))$ and female $(0.66(0.06))$ climbers. ${ }^{1}$ Scores for our subjects exceed some handgrip/mass ratios reported for adult climbers. Mermier et $a l^{18}$ reported grip strength/mass ratios of 0.65 


\section{Take home message}

Although these accomplished young sport climbers have similar anthropometric characteristics to elite adult climbers, it is notable that they perform at very high levels without the severe reductions in body fat that have been reported for adults.

$(0.14)$ and $0.49(0.10)$ for adult male and female recreational climbers respectively. Greater development of forearm musculature in the climbers is suggested by our data that indicate no differences in forearm or forearm + hand volumes compared with controls despite significantly smaller stature and lower body mass.

In summary, high level young competitive climbers present general anthropometric characteristics similar to elite adult climbers. These include relatively small stature, low body mass, low sum of skinfolds, and high handgrip/mass ratio. Relative to age matched athletic non-climbers, climbers also appear to be more linear in body type with narrow shoulders relative to hips. Body composition differences exist between climbers and non-climbing athletes despite equivalent BMI.

\section{ACKNOWLEDGEMENTS}

This study was supported through funding from a 1999/2000 Peter White Scholar Award and the College of Professional Studies of Northern Michigan University. The authors would also like to acknowledge the support of the Junior Competition Climbers Association and Club Sport, Tigard, Oregon for enabling access to the subjects for this study.

\section{Authors' affiliations}

P B Watts, L M Joubert, A K Lish, J D Mast, B Wilkins, Northern Michigan University, Marquette, Ml 49855, USA

\section{REFERENCES}

1 Watts PB, Martin DT, Durtschi S. Anthropometric profiles of elite male and female competitive sport rock climbers. J Sports Sci 1993;11:113-17.
2 Booth J, Marino F, Hill C, et al. Energy cost of sport rock climbing in elite performers. Br J Sports Med 1999;33:14-18.

3 Watts PB, Newbury V, Sulentic J. Acute changes in handgrip strength, endurance, and blood lactate with sustained sport rock climbing. J Sports Med Phys Fitness 1996;36:255-60.

4 Watts PB, Daggett M, Gallagher P, et al. Metabolic responses during sport rock climbing and the effects of active versus passive recovery. Int $J$ Sports Med 2000;21:185-90.

5 Cutts A, Bollen SR. Grip strength and endurance in rock climbers Proceedings of the Institution of Mechanical Engineers 1993;207:87-92. 6 Downing S. Eating disorders: are climbers afflicted? Climbing 1994;15:136-40.

7 Steuart K. Walking a thin line. Rock \& Ice 1996;73:50-1.

8 ÖSK (Öterreichische Sportkletter Kommission). Sportkletterreferat. Langgasse: ÖSK, 1999.

9 Norton K, Olds T, eds. Anthropometrica. Sydney: University of New South Wales Press, 1996.

10 CDC. Growth charts. Centers for Disease Control and Prevention, National Center for Health Statistics, www.cdc/nchg/about/major/ nhanes/growthcharts/charts.html, 2000.

11 Jackson AS, Pollock ML. Practical assessment of body composition. Physician and Sports Medicine 1985;13:75-90.

12 Brozek J, Grande F, Anderson J, et al. Densitometric analysis of body composition: revision of some quantitative assumptions. Ann N Y Acad Sci 1963;110:113-40.

13 Slaughter MH, Lohman TG, Boileau RA, et al. Skinfold equations for estimating body fatness in children and youth. Hum Biol 1988:60:709-23.

14 Billat V, Palleja P, Charlaix T, et al. Energy specificity of rock climbing and aerobic capacity in competitive sport rock climbers. J Sports Med Phys Fitness 1995;35:20-4.

15 Doran DA, Grace SR. Physiological and metabolic responses in novice and recreational rock climbers. In: Messenger N, Patterson W, Brook D, eds. The Science of Climbing and Mountaineering (CD-ROM). Champaign, IL: Human Kinetics Software, 2000.

16 Grant S, Hynes V. Whittaker A, et al. Anthropometric, strength, endurance and flexibility characteristics of elite and recreational climbers. J Sports Sci 1996;14:301-9.

17 Mermier CM, Robergs RA, McMinn SM, et al. Energy expenditure and physiological responses during indoor rock climbing. Br J Sports Med 1997;31:224-8.

18 Mermier CM, Janot JM, Parker DL, et al. Physiological and anthropometric determinants of sport climbing performance. Br J Sports Med 2000;34:359-66.

19 Watts PB, Drobish KM. Physiological responses to simulated rock climbing at different angles. Med Sci Sports Exerc 1998;30:1 11 18-22.

20 Plowman SA, Smith DL. Exercise physiology for health, fitness, and performance. Boston: Allyn and Bacon, 1997.

21 Deurenberg $\mathbf{P}$, Weststrate JA, Seidell JC. Body mass index as a measure of body fatness: age- and sex-specific prediction formulas. $\mathrm{Br} J$ Nutr 1991;65:105-14. 